\title{
Pulmonary embolism in Behcet's disease: a case report
}

\author{
Despoina Moumtzi, Marianna Kakoura \\ Respiratory Department, Papageorgiou General Hospital, Thessaloniki, Greece
}

\begin{abstract}
Behcet's disease (BD) is a vasculitis of unknown etiology. It is often correlated with thrombophilic factors such as V Leiden. Pulmonary involvement is reported in $1-10 \%$ of patients. The most common manifestations are pulmonary aneurysms while pulmonary embolism is a rare complication. A 41-year old man with BD and V Leiden heterozygosity complained of pleurodynia and fever. Pleurodynia deteriorated in the following days and $\mathrm{PE}$ was confirmed by CT angiography, without the presence of aneurysms. After the exclusion of the antiphospholipid syndrome, a therapeutic dose of apixaban was initiated. Two weeks later, pleurodynia relapsed in combination with pleural effusion unilat-
\end{abstract}

Correspondence: Despoina Moumtzi, Respiratory Department,

Papageorgiou General Hospital, Thessaloniki, Greece.

E-mail:dmoumtzi@hotmail.com

Key words: Pulmonary embolism; Behcet's disease; thrombophilia; V Leiden factor.

Conflict of interest: The authors declare that they have no competing interests, and all authors confirm accuracy.

Contributions: All the authors made a substantive intellectual contribution. All the authors have read and approved the final version of the manuscript and agreed to be accountable for all aspects of the work.

Availability of data and materials: All data underlying the findings are fully available.

Ethics approval and consent to participate: No ethical committee approval was required for this case report by the Department, because this article does not contain any studies with human participants or animals. Informed consent was obtained from the patient included in this study

Consent for publication: The patient gave his written consent to use his personal data for the publication of this case report and any accompanying images.

Received for publication: 29 April 2020.

Accepted for publication: 7 September 2020.

${ }^{\circ}$ Copyright: the Author(s), 2020

Licensee PAGEPress, Italy

Monaldi Archives for Chest Disease 2020; 90:1347

doi: 10.4081/monaldi.2020.1347

This article is distributed under the terms of the Creative Commons Attribution Noncommercial License (by-nc 4.0) which permits any noncommercial use, distribution, and reproduction in any medium, provided the original author(s) and source are credited. erally. These findings were attributed to disease exacerbation. For this reason, we decided to enhance the immunosuppressive therapy. Six months later, CTPA showed complete remission of the clots. Vasculitis predisposes to thrombosis with or without coexisting thrombophilia. Clinicians should include them in their differential diagnosis and provide personalized treatment, based on immunosuppressants.

\section{Introduction}

Behcet's disease (BD) is a rare vasculitis characterized by recurrent aphthous mouth ulcers, genital ulcers, uveitis and cutaneous lesions such as erythema nodosum. It is most common in Turkey, where the highest prevalence has been recorded (80-370 cases per 100,000), other Mediterranean countries and in Japan [1]. It usually occurs in young adults aged 20 to 40 years, affecting males 2-5 times more than females [2] Male sex and young age of onset of the disease are associated with a poorer prognosis [1]. The etiology of disease is unknown. Genetic predisposition is considered likely, since it is strongly associated with HLA-B51 [3]. Pulmonary involvement is reported in $1-10 \%$ of patients [2]. The most common manifestations are aneurysms of the pulmonary artery, arterial and venous thrombosis, pulmonary infarctions, recurrent pneumonia, organizing pneumonia and pleurisy [1]. Pulmonary embolism (PE) is a rare complication of the disease. The mechanism of thrombus formation is associated with inflammation of the vessels [4]. Thus, immunosuppressants represent the basis of treatment. Many researchers have suggested a correlation between BD and thrombophilia [5]. One of the most common concomitant factors is the factor $\mathrm{V}$ Leiden. Treatment of thrombotic events in thrombophilic patients with BD is complicated.

\section{Case Report}

Male, 41 years old, active smoker (30 pack/years), was presented to the Emergency Department because of left-sided pleurodynia and fever up to $38.5^{\circ} \mathrm{C}$. He had received clarithromycin for 5 days without relief of symptoms. Clinical examination: temperature $37.5^{\circ} \mathrm{C}, \mathrm{Sp}_{2} 95 \%$, heart rate $79 / \mathrm{min}$, respiratory rate $18 / \mathrm{min}$, blood pressure 110/60 $\mathrm{mmHg}$, lung auscultation: diffuse crackles. The chest X-ray recorded an inflation of the right hilum and linear atelectasis in the middle and lower lung fields. Initial laboratory test: leukocyte count $11.2 \mathrm{X} 10^{3} / \mu \mathrm{l}$ (neutrophils $70 \%$ ), hemoglobin $13.7 \mathrm{~g} / \mathrm{dl}$, platelets $399 \times 10^{3} / \mu \mathrm{l}$, INR 1.17 , D-dimers $3.210 \mathrm{ng} / \mathrm{ml}$, C-reactive protein $10.4 \mathrm{mg} / \mathrm{dl}$, procalcitonin $<0.05 \mathrm{ng} / \mathrm{ml}$.

Personal history: BD diagnosed five years ago, following an event of right lower limb thrombosis with concomitant aphthous mouth and genital ulcers. The patient was positive for HLA-B51 
and V Leiden heterozygosity (PCR-SSP standardization method). His therapy consisted of cyclosporine, colchicine and aspirin. Disease exacerbations with myalgia and arthralgia have been reported and treated with short-term prednisolone and nonsteroidal anti- inflammatory drug.

The patient was treated for pneumonia with ampicillin/sulbactam and azithromycin. A gradual remission of fever was noticed after $48 \mathrm{~h}$; however, the chest X-ray showed possible chronic lesions (Figure 1). The chest computer tomography confirmed our hypothesis: In addition to bronchopulmonary infiltration in the right upper lobe, we also detected, chronic lung lesions typical to the underlying disease. These were pleural effusion, fibrous and atelectatic elements, nodules, ground-glass opacity, and paratracheal lymphadenopathy (Figure 2A).

The symptom of pleurodynia deteriorated in the next days and PE was suspected. Computed tomography pulmonary angiogram (CTPA) was performed and detected thrombi in subfractional branches of the right upper lobe's pulmonary artery (Figure 3). Echocardiography showed no dysfunction of the right ventricle; cardiac enzymes were within normal limits and the compression ultrasonography of the of the lower limb veins was negative for

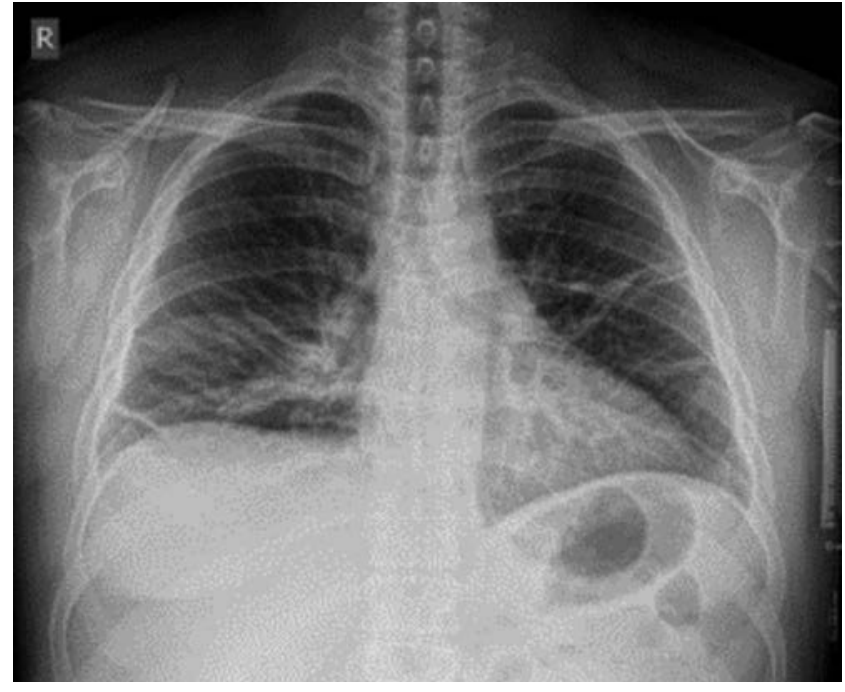

Figure 1. Findings of the chest X-ray: inflation of the right hilum and linear atelectasis in the middle and lower lung fields.


Figure 2. A) Pulmonary manifestation of BD: fibrous and atelectatic elements, nodules, ground-glass opacity. B) Radiological improvement after treatment adaptation, 6 months later. 


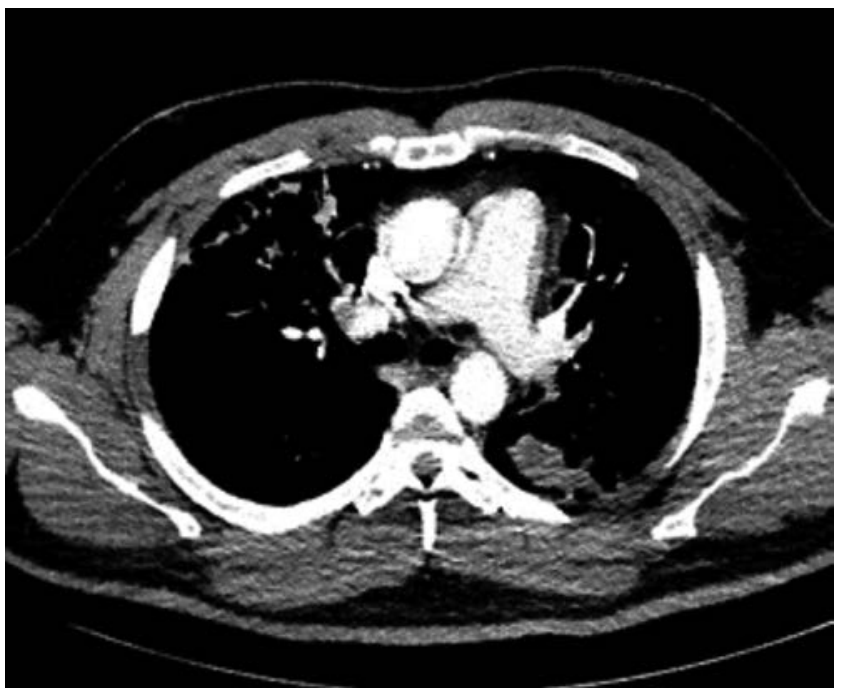

Figure 3. Pulmonary CT angiography: thrombi in subfractional branches of the right upper lobe's pulmonary artery.

clots in the deep venous system. As the presence of aneurysms was excluded, a therapeutic dose of tinzaparin was administered. Antiphospholipid antibodies cardiolipin (ACL-IgG, ACL-IgM) and beta-2 glycoprotein 1 (IgG, IgM) were tested and were negative. After excluding the antiphospholipid syndrome, heparin was replaced by apixaban at a dose of $5 \mathrm{mg}$ twice daily.

Two weeks later the patient presented to the Emergency Department complaining of right-sided pleurodynia. Chest X-ray detected a right-sided pleural effusion, which did not exist before. The effusion was confirmed by lung ultrasounds and was minimal and non-puncturable. The patient's imaging was regarded as disease exacerbation. As suggested by latest guidelines [6,7] methylprednisolone $1 \mathrm{mg} / \mathrm{kg} / \mathrm{h}$, was administered for 3 days with gradual decrease. It was also decided to enhance the immunosuppressive therapy with colchicine $1 / 2 \mathrm{mg}$ daily. After 7 days of hospitalization, the remission of pleural effusion was confirmed by ultrasounds. The patient was discharged on instructions for a maintenance dose of prednisolone, cyclosporine, colchicine, and a therapeutic dose of apixaban. In a re-examination 6 months later the patient was in excellent clinical condition and the CTPA showed complete remission of the multiple radiological lesions and filling defects (Figure 2B).

\section{Discussion}

Symptoms of persistent pleurodynia, fever and dyspnea are usual in patients with BD. In addition to infection, other causes are exacerbation of the disease, pulmonary artery aneurysm and, rarely, pulmonary embolism $[8,9]$. While in a patient without BD we would consider a PE or infection, in a patient with $\mathrm{BD}$ we must rule out the presence of pulmonary artery aneurysm (PAA) [10], which are a characteristic complication of the disease, especially in young men. PAA may be multiple and have high rates of recurrence [2]. They are more frequent than pulmonary embolism. BD is often diagnosed after the detection of PAA is in a patient. PAA have a poor prognosis, especially when the diameter is more than $3 \mathrm{~cm}$. It is estimated that
$30 \%$ of BD patients with PAA die within 2 years. Hemoptysis (sometimes massive) is often the first clinical sign [8]. In this case, emergency treatment with open surgery or embolism is the only choice. Otherwise, invasive methods are recommended in isolated PAA. Also it is important that enhanced immunosuppressive therapy is preceded, as it seems to prevent postoperative complications such as pseudoaneurysms and bronchopulmonary arterial fistulas $[6,11]$. Therefore, the existence of possible hidden PAA should be checked radiologically by Computed Tomography angiography or Magnetic Resonance angiography before initiating any treatment, as in the case reported. In addition, it is usual to detect clots within PAA, or even coexistence of pulmonary arterial thrombosis, aneurysms and deep vein thrombosis (Hughes-Stain syndrome) [2]. In this case, the use of anticoagulants is controversial, since there is an increased risk of bleeding and even fatal hemoptysis. Many studies show that immunosuppressive therapy combined with corticosteroids results in remission and even disappearance of PAA [3]. In particular, treatment involves cyclophosphamide (orally $2 \mathrm{mg} / \mathrm{kg} /$ day or monthly 1 $\mathrm{g}$ intravenous) combined with tapered methylprednisolone (starting dosage $1 \mathrm{mg} / \mathrm{kg}$ ) for at least one year after complete remission. Alternatively, azathioprine (orally $2.5 \mathrm{mg} / \mathrm{kg} / \mathrm{day}$ ) in place of cyclophosphamide, is equally effective in the remission of PAA [6].

Administration of anticoagulants is considered by many researchers with skepticism because of the different mechanism of thrombosis in the BD [11]. It is an in situ thrombosis as the result of vasculitis [2]. Inflammation affects all layers of the vessels and leads to diffuse endothelial cell injury, which is the key factor that predisposes to thrombosis. Many researchers associate the endothelial cell injury with subsequent inhibition of natural anticoagulants (reduced thrombomodulin, which is an endothelial derivate) and release of endothelial derivatives such as the von Willebrand factor, which results in platelet release, and plasminogen inhibitor-1 (PAI-1), which inhibits the mechanism of fibrinolysis [12].

In non-extensive PE, the use of antiplatelet agents was regarded sufficient in the past [3]. According to recent data, the presumption that arterial and venous thrombosis are distinct entities is rebutted, since researchers who analyzed the red clot of venous thrombosis and the white clot of arterial thrombosis detected a common network of fibrin, erythrocytes, platelets and leukocytes [13]. Therefore, platelets known to be responsible for arterial thrombosis also appear to be associated with the pathogenesis of venous thrombosis. As a result, patients with arterial thrombosis have a high risk for venous thrombosis. This coexistence is present in many diseases such as BD [13]. There are no studies that directly compare anticoagulants with antiplatelets, and the choice should be individualized in each case, taking into account the advantages and disadvantages of each drug. Thus, if there is any indication of pulmonary vasculitis, increased bleeding risk and suspicion of hidden aneurysms, anticoagulants are contraindicated [14]. On the other hand, antiplatelets do not present such a high bleeding risk and have beneficial properties on the aortic wall. In particular, they were found to reduce inflammation of the aorta and stabilize the aortic wall in abdominal aortic aneurysms, preventing a potential rupture [14]. Furthermore, the International Collaboration of Aspirin Trials for Current Venous Thromboembolism (INSPIRE) found that for patients with first unprovoked venous thromboembolism aspirin reduces risk of recurrence by more than a third, unlike anticoagulants [15]. On the other hand, non-use of anticoagulant drug was found to increase the risk for post-thrombotic syndrome. In addition, the clinical significance of subsegmental PE is questionable [16]. These patients are usually hemodynamically stable and asymptomatic. They also have decreased rate of recurrent VTE [17]. In this case many researchers suggest no anticoagulation with close monitoring [18]. However, our 
patient had symptomatology (pleurodynia) and was diagnosed with PE while already receiving aspirin and immunosuppressive treatment with cyclosporine. These led to the decision to initiate anticoagulation treatment.

In $\mathrm{BD}$ cases many researchers observed a deficiency of natural inhibitors of coagulation such as proteins $\mathrm{C}, \mathrm{S}$ and antithrombin. A combination of factors such as endothelial injury, hyperhomocysteinemia and elevated plasma levels of endothelial derivatives, such as vWF and tissue plasminogen activator (tPA), has also been observed [12]. Factor V Leiden has been isolated in $29 \%$ of BD patients and $37.5 \%$ of $\mathrm{BD}$ patients with thrombosis [19]. It is the most common inherited thrombophilia, with a prevalence of $3 \%$ to $7 \%$ in the general US population. The question that arises is whether thrombophilia affect the incidence of PE in BD patients. Factor V Leiden seems to increase the risk of thrombosis but at a rate similar to that of healthy population [5]. Martinelli et al. found low rates of prevalence of V Leiden in PE cases, at the rate of $4.9 \%$ [20], while Turkstra et al. at the rate of 7.4\% [21]. Also, its presence is mainly associated with DVT and not with PE [19,21]. Specifically, Manten et al. [19] found that the relative risk for PE in $\mathrm{V}$ Leiden heterozygotic patients was 3 to 4 times greater than control group, while for DVT was 7 times greater. Moreover, Turkstra et al. conclude that prevalence of factor $\mathrm{V}$ Leiden in patients with PE was $50 \%$ lower than the prevalence in patients with DVT [21]. A series of studies failed to correlate thrombotic events in BD patients with factor V Leiden, such as Silingardi et al. [22] and Caramaschi et al. [23] in Italian patients and Ricart et al. [24] in Spanish patients. Chamorro et al support that factor V Leiden is responsible for thrombotic events, but only in Turkish patients with BD [25]. Finally Leiba et al. suggest that hypertrigyceridaimia instead of thrombophilia might be a risk factor for thrombosis in BD [26].

\section{Conclusions}

In view of the above, we conclude that $\mathrm{BD}$ as a vasculitis predisposes to thrombotic events with or without co-existing thrombophilia, the treatment of which should be personalized. The new guidelines on PE correctly include autoimmune diseases among the risk factors for thrombosis [27]. For these reasons, clinicians should include them in their differential diagnosis.

\section{References}

1. Erkan F, Gül A, Tasali E. Pulmonary manifestations of Behçet's disease. Thorax 2001;56:572-8.

2. Hiller N, Lieberman S, Chajek-Shaul T, et al. Thoracic manifestations of Behçet disease at CT. Radiographics 2004;24:801-8.

3. Erkan F, Kyan E, Tunac A. Pulmonary complications of Behçet's disease. Clin Chest Med 2002;23:493-503.

4. Kim JH, Lee KA, Jung MY, et al. Pulmonary thromboembolism in patient with coexistence of Behçet's disease and antiphospholipid syndrome. Int J Rheum Dis 2018;21:2188-92.

5. Seyahi E, Yurdakul S. Behçet's syndrome and thrombosis. Mediterr J Hematol Infect Dis 2011;3:e2011026.

6. Hatemi G, Christensen R, Bang D, et al. 2018 Update of the EULAR recommendations for the management of Behçet's syndrome. Ann Rheum Dis 2018;77:808-18.

7. Hatemi G, Seyahi E, Fresko I, et al. One year in review 2018:
Behçet's syndrome. Clin Experim Rheumatol 2018;36:13-27.

8. Seyahi E, Melikoglu M, Akman C, et al. Pulmonary artery involvement and associated lung disease in Behçet disease: A series of 47 patients. Medicine (Baltimore) 2012;91:35-48.

9. Haskard DO, Ambrose N L. Differential diagnosis and management of Behçet syndrome. Nat Rev Rheumatol 2013;9:79-89.

10. Edrees A, Naguib S, El Menyawi M, et al. Pulmonary manifestations in a group of patients with Behcet's disease. Int J Rheum Dis 2017;20:269-75.

11. Uzun O, Akpolat T, Erkan L. Pulmonary vasculitis in Behçet disease: A cumulative analysis. Chest 2005;127:2243-53.

12. Leiba M, Sidi Y, Gur H, et al. Behçet's disease and thrombophilia. Ann Rheum Dis 2001;60:1081-5.

13. Becatti M, Emmi G, Bettiol A. Behçet's syndrome as a tool to dissect the mechanisms of thrombo-inflammation: clinical and pathogenetic aspects. Clin Experim Immunol 2019;195:322-33.

14. Merashli M, El Eid R, Uthman I. A review of current management of vasculo-Behcet's. Curr Opin Rheumatol 2018 30:50-6.

15. Simes J, Becattini C, Agnelli G, et al. Aspirin for the prevention of recurrent venous thromboembolism: the INSPIRE collaboration. Circulation 2014;130:1062-71.

16. Peiman S, Abbasi M, Allameh S, et al. Subsegmental pulmonary embolism: A narrative review. Thromb Res 2016;138:55-60.

17. Carrier M, Klok F. Symptomatic subsegmental pulmonary embolism: to treat or not to treat? Hematology Am Soc Hematol Educ Program 2017;2017:237-41.

18. Carrier M, Righini M, Le Gal G. Symptomatic subsegmental pulmonary embolism: what is the next step? J Thromb Haemost 2012; 10:1486-90.

19. Manten B, Westendorp RGJ, Koster T, et al. Risk factor profiles in patients with different clinical manifestations of venous thromboembolism: A focus on the factor V Leiden mutation. Thromb Haemost 1996;76:510-3.

20. Martinelli I, Cattaneo M, Panzeri D, Mannucci PM. Low prevalence of factor V:Q506 in 41 patients with isolated pulmonory embolism. Thromb Haemost 1997;77:440-3.

21. Turkstra F, Karemaker R, Kuijer PM, et al. Is the prevalence of the factor $\mathrm{V}$ Leiden mutation in patients with pulmonary embolism and deep vein thrombosis really different? Thromb Haemost 1999;81:345-8.

22. Silingardi M, Salvarani C, Boiardi L, et al. Factor V Leiden and prothrombin gene G20210A mutations in Italian patients with Behçet's disease and deep vein thrombosis. Arthr Care Res 2004;51:177-83.

23. Caramaschi P, Poli G, Bonora A, et al. A study on thrombophilic factors in Italian Behcet's patients. Joint Bone Spine 2010;77:330-4.

24. Ricart JM, Vayá A, Todoli J, et al. Thrombophilic risk factors and homocysteine levels in Behęt's disease in eastern Spain and their association with thrombotic events. Thromb Haemost 2006;95:618-24.

25. Chamorro AJ, Marcos M, Hernández-García I, et al. Association of allelic variants of factor V Leiden, prothrombin and methylenetetrahydrofolate reductase with thrombosis or ocular involvement in Behçet's disease: A systematic review and meta-analysis. Autoimmun Rev 2013;12:607-16.

26. Leiba M, Seligsohn U, Sidi Y, et al. Thrombophilic factors are not the leading cause of thrombosis in Behçet's disease. Ann Rheum Dis 2004;63:1445-9.

27. Konstantinides SV, Meyer G, Becattini C, et al. 2019 ESC Guidelines for the diagnosis and management of acute pulmonary embolism developed in collaboration with the European Respiratory Society (ERS). Eur Heart J 2020;41:543-603. 